Gut, 1987, 28, 721-725

\title{
Evaluation of the hydrogen breath test in man: definition and elimination of the early hydrogen peak
}

\author{
G MASTROPAOLO AND W D W REES \\ From the Department of Gastroenterology, Hope Hospital Salford
}

\begin{abstract}
SUMmARY After ingestion of a non-absorbable carbohydrate breath hydrogen excretion increases early at about 10 minutes, and again later when the ingested carbohydrate enters the caecum. The late rise has been used as a marker of mouth to caecum transit time, but the source of the early rise has not been satisfactorily explained. We studied in 60 healthy volunteers the source and frequency of the early rise in breath hydrogen after ingestion of a non-absorbable carbohydrate. After ingestion of either lactulose solution $(10 \mathrm{~g}$ in $150 \mathrm{ml}$ water), lentil soup ( $46 \mathrm{~g}$ carbohydrate) or solid meal containing baked beans ( $15 \mathrm{~g}$ carbohydrate), breath hydrogen was significantly raised above basal concentrations within 10 minutes $(81 \pm 27,395 \pm 138$ and $110 \pm 52 \%$ above basal respectively). A significant rise in breath hydrogen $(75 \pm 21 \%$ ) occurred 10 minutes after sham lactulose feeding (lactulose applied to oral cavity but not swallowed), but no early peak occurred after sham saccharin feeding (non-fermentable carbohydrate), intragastric or intraduodenal administration of lactulose. Ten of the 12 subjects given lactulose sham feeding were restudied after oral hygiene with chlorhexidine mouthwash. In these the early hydrogen peak was abolished. Oral hygiene also reduced the occurrence and magnitude of the early hydrogen rise after lactulose ingestion. These findings indicate that the early rise in breath hydrogen observed after ingestion of lactulose is produced by interaction with oral bacteria.
\end{abstract}

The hydrogen breath test is a simple, inexpensive and non-invasive method for estimating small bowel transit time,' 2 detecting the existence of excess bacteria in the small intestine, ${ }^{3+}$ and demonstrating carbohydrate maldigestion or malabsorption. ${ }^{56}$

Breath hydrogen is derived from the interaction between ingested carbohydrate and intestinal bacteria. In studies of intestinal transit, where a nonabsorbable carbohydrate is used, and in carbohydrate malabsorption the interaction occurs in the proximal large bowel. Transit time is calculated from the interval between meal ingestion and the detection of a significant and sustained rise in breath hydrogen excretion.' ' The transit time, however, merely reflects arrival of the 'head' of the meal in the caecum and is a crude measure of whole meal transit through

Address for correspondence: Dr W D W Rees. Hope Hospital, Salford M6 8HD Received for publication 6 Octoher. 1986. the small intestine. In small intestinal bacterial overgrowth states the interaction occurs within the jejunum or ileum resulting in hydrogen peak before the carbohydrate enters the caecum. ${ }^{3+}$ In a number of studies, however, ${ }^{7-4}$ the existence of an early rise in breath hydrogen, unrelated to bacterial contamination of the upper gut, has been shown, about 10 to 20 minutes after meal ingestion. This early rise is not caused by the rapid transit of a component of the meal into the colon." Until recently, it had been accepted that this early peak was caused by a gastroileal reflex clearing any undigested carbohydrate from the terminal ileum into the caecum and mixing it with colonic bacteria. ${ }^{7 \times}$ Recent studies, however, have provided evidence that interaction between oral bacteria and ingested carbohydrate may be partly responsible for the early rise in breath hydrogen. ${ }^{411}$ We have investigated this possibility further in healthy volunteers. 


\section{Methods}

SUBJECTS

The studies were carried out on 60 healthy volunteers aged between 18 and 46 years (mean 23.4 years). There were 27 men and 33 women. The experiments had been approved by the Salford District Ethical Committee and each subject provided informed consent.

MEASUREMENT OF BREATH HYDROGEN

After a 14 hour fast, basal breath hydrogen levels were determined by collecting $20 \mathrm{ml}$ aliquots of the end expiratory volume at 10 minute intervals. The hydrogen concentration (parts per million) was measured by means of an electrochemical breath analyser (GMI Medical Ltd, UK) which was calibrated using samples of room air (undetectable hydrogen) and a standard gas mixture containing 96 ppm of hydrogen. Repeated measurements on a gas sample produced only $4 \%$ variation. Calibration of the breath analyser was checked in frequent intervals (every 10 samples) during each study.

\section{EXPERIMENT DESIG N \\ Study of breath hydrogen profiles after different test meals}

In three groups of 12 subjects breath hydrogen concentration was studied after administration of three test meals: $150 \mathrm{ml}$ of $7 \%$ lactulose solution $(10.5 \mathrm{~g}$ lactulose in water), $300 \mathrm{ml}$ lentil soup (300 calories, $13 \mathrm{~g}$ protein, $36 \mathrm{~g}$ carbohydrate, $11 \mathrm{~g}$ fat) containing $10.5 \mathrm{~g}$ lactulose, and a solid meal consisting of two scrambled eggs and $150 \mathrm{~g}$ baked beans $(15 \mathrm{~g}$ non-absorbable carbohydrate and 300 calories). Breath hydrogen concentration was recorded at 10 minute intervals during a 30 minute fasting period and after meal ingestion until a sustained rise in breath hydrogen occurred signalling arrival of carbohydrate in the caecum.

\section{Source of the early hydrogen peak}

Having defined the existence of an early hydrogen peak after ingestion of fermentable carbohydrate, a further four series of experiments were conducted to determine its source. One group of six subjects were studied on three occasions after $150 \mathrm{ml}, 60 \mathrm{ml}$, and 30 $\mathrm{ml}$ lactulose solution ( $7 \%$ solution) was instilled directly into the stomach through a 9 FG nasogastric tube. A second group of 12 subjects had $60 \mathrm{ml}$ of $7 \%$ lactulose instilled direct into the duodenum over a five minute period to simulate early gastric emptying after ingestion of $150 \mathrm{ml}$ of $7 \%$ lactulose. The third group of 12 volunteers was studied on two separate occasions in randomised order. On one occasion 30 $\mathrm{ml}$ of $7 \%$ lactulose solution was retained in the mouth for one minute and expelled, while on the second occasion a similar volume of $1 \%$ saccharin solution was used. In the final group of 20 volunteers, oral hygiene with $0.2 \%$ chlorhexidine mouthwash was conducted before administration of either sham lactulose ( $30 \mathrm{ml}$ of a $7 \%$ solution) in 10 subjects ( 10 of the 12 subjects given sham lactulose in group 3 ) or $150 \mathrm{ml}$ of ingested lactulose solution $(7 \%)$ in 10 subjects. The hygiene procedure consisted of rinsing the mouth over two minutes with two aliquots of 20 $\mathrm{ml} 0 \cdot 2 \%$ chlorhexidine solution, followed by a tap water rinse. In all these studies, breath hydrogen was measured at 10 minute intervals for 30 minutes before and 40 minutes after administration of the test solutions.

\section{STATISTICAL ANALYSIS AND EXPRESSION OF RESULTS}

Results are expressed as parts per million of breath hydrogen or per cent change in "basal breath hydrogen' and differences between mean values were assessed using the Student's $t$ test for paired variables. In studies on the early peak 'basal breath hydrogen' represented levels after a 14 hour fast while in mouth to caecum transit time measurements 'basal breath hydrogen' represented levels between termination of the early peak and onset of the secondary rise. Mouth to caecum transit time (minutes) was therefore calculated from the interval between ingestion of a test solution and a $3 \mathrm{ppm}$ increase in "basal breath hydrogen' which persisted for at least 30 minutes."

\section{Results}

A total of 130 hydrogen breath tests were carried out on 60 subjects. Fasting breath hydrogen in these studies ranged from 1 to $85 \mathrm{ppm}$ with a mean and standard error of $13.8 \pm 1.5 \mathrm{ppm}$.

BREATH HYDROGEN PROFILES AFTER DIFFERENT TEST MEALS

After ingestion of lactulose solution, lentil soup and the solid meal the pattern of breath hydrogen elevation was similar and consisted of an early rise followed by a later and more sustained increase, signalling arrival of carbohydrate in the caecum. In 34 of the 36 subjects an early peak was demonstrable within 10 minutes of meal ingestion. After lactulose ingestion, the breath hydrogen during the early peak increased from fasting levels of $10 \pm 2 \mathrm{ppm}$ to $17 \pm 3$ ppm $(73 \cdot 1 \pm 24 \cdot 3 \%$, Figure $1, \mathrm{p}<0 \cdot 05)$. The increase after the solid meal was $17 \pm 4 \mathrm{ppm}$ to $25 \pm 5 \mathrm{ppm}$ $(110.1 \pm 52.5 \%, p<0.05)$ and the lentil soup, $11 \pm 3$ ppm to $23 \pm 4$ ppm $(395.0 \pm 137.9 \%, p<0.05)$ (Fig. 1). Hydrogen levels subsequently decreased, reaching basal values $35 \cdot 0 \pm 4 \cdot 3$ minutes after the lactulose 


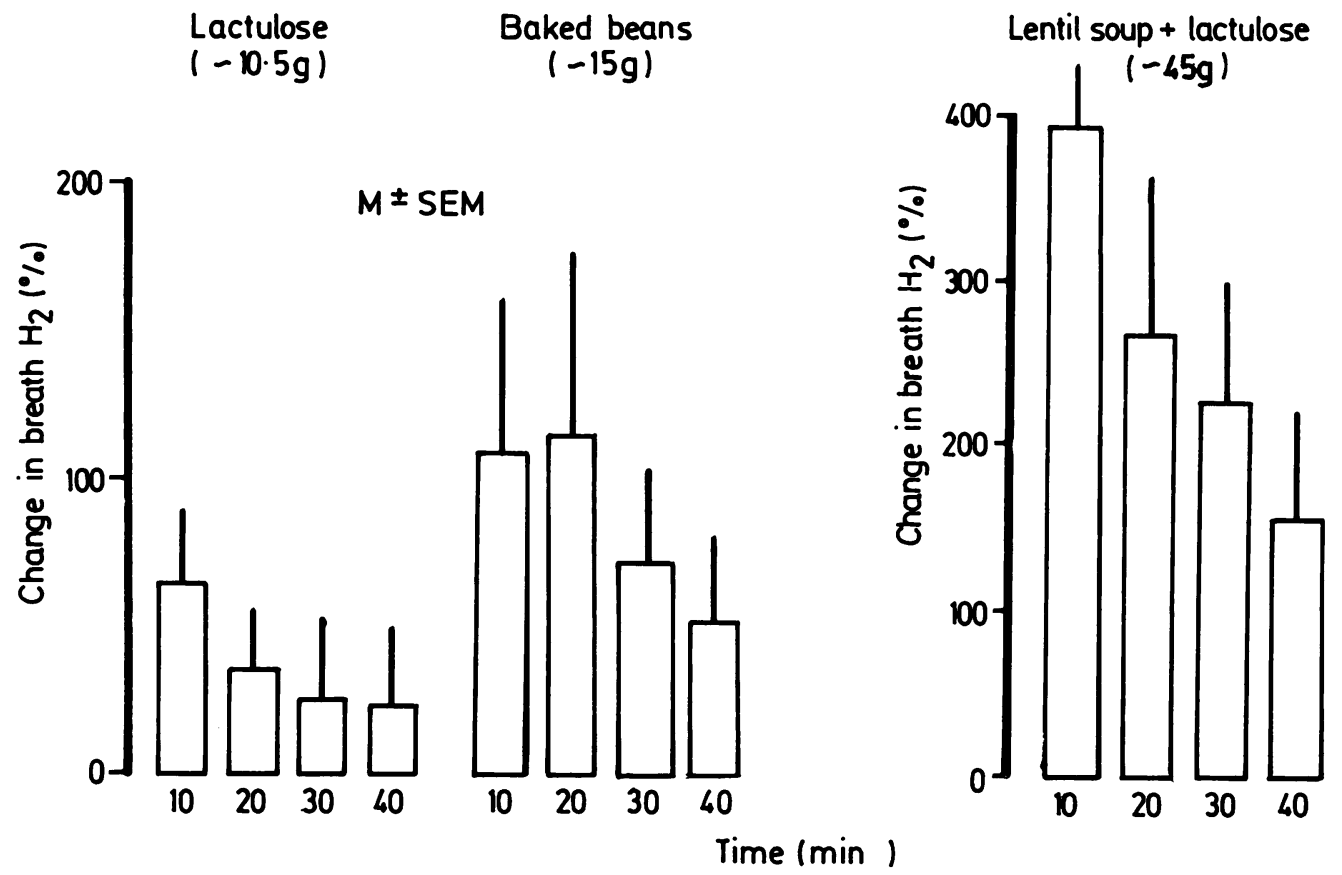

Fig. 1 Per cent change in basal breath hydrogen over a 40 minute period after ingestion of lactulose solution, solid meal or lentil soup. Values represent means $\pm S E$ of 12 studies.

solution, $73 \cdot 3 \pm 6 \cdot 2$ minutes after the solid meal and $58 \cdot 0 \pm 5 \cdot 3$ minutes after lentil soup. In the remaining two subjects, one in the lactulose solution group and the other in the solid meal group, smaller increases in hydrogen $(5 \cdot 2$ and $36 \%$ of basal) were observed within 20 minutes of ingestion. Although the magnitude of the early peak appeared proportional to the carbohydrate load in the three meals, this correlation did not achieve statistical significance. The secondary sustained peak occurred $88 \cdot 7 \pm 9 \cdot 1$ minutes after ingesting the lactulose meal, $100 \cdot 5 \pm 7 \cdot 2$ minutes after lentil soup and $347 \cdot 0 \pm 33 \cdot 2$ minutes after the solid meal. These values indicate the mouth to caecum transit times for the three meals. Thirty minutes after the first arrival of the meals at the caecum, the per cent increase in basal breath hydrogen (recorded immediately before the secondary sustained rise) was $309 \cdot 9 \pm 47 \cdot 1$ after lactulose, $112 \cdot 0 \pm 29 \cdot 5$ after the solid meal and $631 \cdot 0 \pm 239 \cdot 6$ after the lentil soup.

SOURCE OF THE EARLY PEAK

After intragastric and intraduodenal administration of lactulose, no early rise in breath hydrogen was observed. The $60 \mathrm{ml}$ bolus data are illustrated in Figure 2. In these experiments, the 'secondary' rise in breath hydrogen was observed 109.1 \pm 6.9 minutes after intragastric lactulose and $39 \cdot 3 \pm 6 \cdot 8$ minutes after intraduodenal lactulose; hydrogen levels being $461 \cdot 3 \pm 102 \cdot 1 \%$ and $412 \cdot 3 \pm 194 \cdot 4 \%$ above basal values (recorded immediately before the sustained secondary rise) respectively.

Eleven of the 12 subjects after sham lactulose feeding showed a rise in breath hydrogen within 10

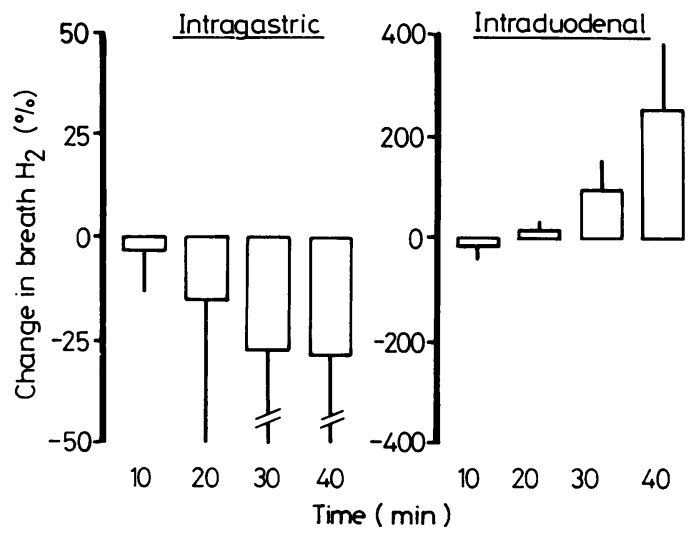

Fig. 2 Percent change in basal breath hydrogen after intragastric and intraduodenal administration of lactulose $(60 \mathrm{ml})$. Values over the 40 minute periods represent means $\pm S E$ of six subjects for the intragastric study and 12 for the intraduodenal study. 


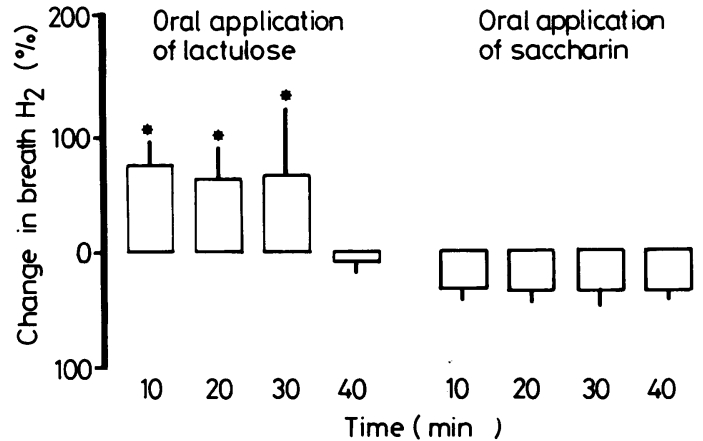

Fig. 3 Per cent changes in basal breath hydrogen after sham feeding with either lactulose or saccharin. Values represent means $\pm S E$ of 12 subjects. ${ }^{*}$ Represents statistical significance of $p<0.01$ when the paired variables were compared after lactulose and saccharin.

minutes (Fig. 3). The increase was from $9 \pm 2$ to $13 \pm 2 \mathrm{ppm}(75 \cdot 1 \pm 21 \cdot 0 \%)$ and its duration $28 \cdot 6 \pm 3 \cdot 3$ minutes. In contrast after saccharin sham feeding there was no rise in breath hydrogen (Fig. 3).

After application of the chlorhexidine mouthwash lactulose sham feeding did not result in an early rise in breath hydrogen (Fig. 4), the magnitude of the change in breath hydrogen being significantly less than in control experiments $(-6 \cdot 7 \pm 11.4 \%$ compared with $75 \cdot 1 \pm 21 \cdot 0 \%, \mathrm{p}<0 \cdot 02, \mathrm{n}=10$ ). When oral hygiene preceded ingestion of a lactulose solution, an early rise in breath hydrogen occurred in six of 10 subjects, but its magnitude was significantly less than in control experiments $(18 \cdot 1 \pm 13 \cdot 3 \%$ compared with $73 \cdot 1 \pm 24 \cdot 3 \%, \mathrm{p}<0 \cdot 05, \mathrm{n}=10)$.

\section{Discussion}

Although not documented in early publications on the hydrogen breath test, ${ }^{135}$ the existence of an early rise in breath hydrogen after carbohydrate ingestion has recently been described..$^{710}$ This was initially attributed to a gastroileal reflex resulting in the delivery of carbohydrate (undigested food residue and mucus) into the caecum and subsequent fermentation by colonic bacteria. ${ }^{7 \times}$ The early peak is not caused by rapid transit of a component of the meal and the transient nature of the early peak is not consistent with fermentation by small intestine bacteria. ${ }^{3+7}$

In a recent study, Read et al ${ }^{4}$ examined the source of the early rise in breath hydrogen after ingestion of a meal containing baked beans. The incidence of an early peak in their study $(89 \%)$ was similar to that in ours using three different meals $(94 \%)$. The magnitude of the early rise was also similar $(110 \%)$ considering that our subjects ingested $150 \mathrm{~g}$ of beans

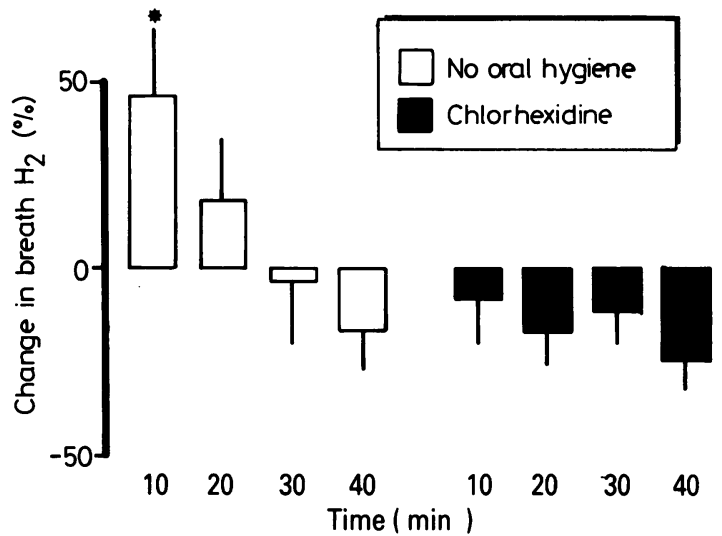

Fig. 4 Change in basal breath hydrogen after sham feeding with lactulose, before and after oral hygiene with chlorhexidine. ${ }^{*}$ Represents statistical significance at the level of $p<0.05$ when the 10 minute paired variables were compared. Values represent the means $\pm S E$ of 10 subjects.

compared with $120 \mathrm{~g}$ in Reads' study. Oral application of $50 \%$ lactulose solution in nine subjects produced an immediate rise in breath hydrogen $(200 \%$ above basal) which was significantly reduced by oral hygiene. This response occurred within minutes of administration but its duration was not monitored for longer than five minutes. Thompson et al " showed a similar rise in breath hydrogen after oral application of $20 \%$ sucrose. The timing of the rise, peak and fall in breath hydrogen after sucrose was very similar to that observed in our study using $7 \%$ lactulose solution. The three studies confirm that oral fermentation of carbohydrate does occur and may contribute to the early rise in breath hydrogen.

Read et al, ${ }^{,}$however, calculated from their studies that buccal fermentation was unlikely to be the major source of the early hydrogen peak after ingestion of a solid meal. Although no actual data were presented in their article, ${ }^{y}$ the authors claimed that oral hygiene eight minutes after starting ingestion of a solid meal containing baked beans, did not significantly influence the resulting early hydrogen peak. They also observed that the magnitude of the early peak was greater if subjects had eaten unabsorbable carbohydrate on the evening before study. It was therefore suggested that the majority of the early rise was devised from a gastroileal reflex delivering unabsorbed carbohydrate into the colon immediately after meal ingestion. The authors, however, did not study the effect of oral hygiene on the hydrogen profile obtained after lactulose ingestion.

We have therefore studied the source of the early hydrogen rise further. Our results show that the onset of the rise is not influenced by the type of carbohydrate 
ingested or its physical nature. Infusion of lactulose directly into the stomach or duodenum, however, failed to elicit an early rise suggesting that either contact between lactulose and mucosal proximal to the stomach is essential for its generation or that oral lactulose induces a 'cephalic' response inducing ileal emptying of unabsorbed carbohydrate into the caecum. Oral application of saccharin, a nonfermentable sweetening agent, failed to produce an early rise in breath hydrogen and this suggests that a cephalic response to ingested sugar is an unlikely mechanism for generating the early peak. These findings thus suggest that buccal fermentation plays an important role in causing the early peak in breath hydrogen after lactulose ingestion. This is further supported by our confirmation that oral hygiene abolishes the rise in breath hydrogen produced by buccal application of lactulose and that it significantly reduces the early peak produced by ingestion of a lactulose solution.

It is conceivable that different carbohydrates both absorbable and non-absorbable, produce an early rise in breath hydrogen by different mechanisms. We have not studied the effect of oral hygiene on the hydrogen rise produced by lentil soup or baked beans. The observations of Read et al ${ }^{4}$ suggest that baked beans produce the early rise by a gastroileal reflex but the timing of their oral hygiene was different from our study and it would be of interest to repeat this experiment, using oral hygiene before meal ingestion. It would also be of interest to evaluate whether the physical nature of carbohydrate influences buccal fermentation as there is no evidence that oral bacteria are capable of interacting with the carbohydrate in food such as baked beans.

Our observations suggest that buccal fermentation of lactulose is a major component of the early rise in breath hydrogen. Care should therefore be taken to ensure meticulous oral hygiene before using such carbohydrate solutions to evaluate small bowel function by means of breath hydrogen analysis. Although our results are applicable only to the use of lactulose in measuring intestinal transit or detecting bacterial overgrowth, similar precautions should be taken when using other carbohydrates until conclusive data are available that they do not generate hydrogen by buccal fermentation.

The authors wish to thank Professor L A Turnberg for his advice and encouragement, Ms J Rostron for typing the manuscript, the Department of Medical Illustration, Hope Hospital for preparing the figures, and the Wellcome Trust for supporting Dr G Mastropaolo.

\section{References}

1 Bond $\mathrm{JH}$, Levitt MD. Investigation of small bowel transit time in man utilising pulmonary hydrogen $\left(\mathrm{H}_{2}\right)$ measurements. J Lab Cell Med 1975; 84: 546-55.

2 La Brooy SJ, Male MS, Beavis BK, Misiewicz JJ. Assessment of the reproducibility of the lactulose $\mathrm{H}_{2}$ breath test as a measure of mouth to caecum transit time. Gut 1983; 24: 893-6.

3 Metz G, Gassul MA, Deason BS, Senkings DSA, Blendis LM. Breath hydrogen test for small intestine bacterial colonization. Lancet 1976; i: 668-9.

4 Rhodes JM, Middleton O, Jewell DP. The lactulose hydrogen breath test as a diagnostic test for small bowel overgrowth. Scand J Gastroenterol 1979; 14: 333-6.

5 Bond JH, Levitt MD. Quantitative measurement of lactulose absorption. Gastroenterology 1979; 70: 1058-62.

6 Metz G, Jenkins DJA, Newman A, Blendis LM. Breath hydrogen in hyposucrosia. Lancet 1976; i: 119-20.

7 Read NW, Miles CA, Fisher D, et al. Transit of a meal through the stomach, small intestine and colon in normal subjects and its role in the pathogenesis of diarrhoea. Gastroenterology 1980; 79: 1276-82.

8 Kerlin P, Phillips S. Differential transit of liquids and solid residue through the human ileum. Am J Physiol 1983; 245: G38-G43.

9 Read NW, Al-Janabi MN, Bates TE, et al. Interpretation of the breath hydrogen profile obtained after ingesting a solid meal containing unabsorbable carbohydrate. Gut 1985; 26: 834-42.

10 Thompson DG, Binfield P, De Belder A, O'Brien J, Warren NA, Wilson M. Extraintestinal influences on exhaled breath hydrogen measurements during the investigation of gastrointestinal disease. Gut 1985; 26: 1349-52. 